306

A NOVEL ANTI-OXIDANT TO COUNTERACT OXIDATIVE STRESS DURING RESUSCITATION AFTER BIRTH ASPHYXIA

doi:10.1136/archdischild-2012-302724.0306

${ }^{1} \mathrm{E}$ Henckel, ${ }^{2} \mathrm{R}$ Solberg, ${ }^{2} \mathrm{TE}$ Calisch, ${ }^{3} \mathrm{~S}$ Norgren, ${ }^{1} \mathrm{~K}$ Bohlin, ${ }^{2} \mathrm{OD}$ Saugstad. 'Department of Clinical Science, Intervention and Technology, Karolinska Institutet, Stockholm, Sweden; ${ }^{2}$ Department of Pediatric Research, Oslo University Hospital - Rikshospitalet, Oslo, Norway; ${ }^{3}$ Department of Women and Child Health, Karolinska Institutet, Stockholm, Sweden

Background and Aims Oxidative stress contributes to tissue damage after perinatal asphyxia. The thiol-containing free radical scavenger $N$-acetylcysteine amide (NACA) is a promising new antioxidant with good penetration into the mitochondria. The objective was to investigate the protective effect of NACA in a piglet model of birth asphyxia.

Methods Anesthetized newborn piglets ( $n=51$ ) were subjected to global hypoxemia and block-randomized to either

1. intravenous administration of NACA $300 \mathrm{mg} / \mathrm{kg}$ and resuscitated with $21 \%$ oxygen for $30 \mathrm{~min}$,

2. saline and $21 \%$ oxygen,

3. NACA and $100 \%$ oxygen or

4. saline and $100 \%$ oxygen.

After resuscitation, the piglets were followed for 9 hours and samples for several markers of injury and oxidative stress were collected. Reported here are clinical parameters and measurements of reduced to oxidized gluthatione (GSH/GSSG).

Results Thirty minutes after end-resuscitation metabolic acidosis was less pronounced in the 100\%-NACA group compared to $100 \%$-oxygen-alone (lactate $8.1 \pm 2.6$ vs $10.9 \pm 3.4$, $\mathrm{p}<0.05$ ). This difference was not shown for the $21 \%$-oxygen groups. Mean arterial blood pressure and hemoglobin levels remained similar between the groups. The GSSG values were generally low. At end-resuscitation GSH was lower in $100 \%$-NACA compared to $100 \%$-oxygen-alone group (164 \pm 111 vs $255 \pm 113 \mu \mathrm{mol}, \mathrm{p}<0.05)$ and delta-GSH during resuscitation greater $(143 \pm 49$ vs $32 \pm 66 \mu \mathrm{mol} p<0.001)$.

Conclusions The data indicate that NACA may enhance immediate recovery, improve mithochondrial glutatione metabolism and restore the cell to a normal metabolism following asphyxia and rescucitation. Upcoming analyses of histopathology and injury markers will further elucidate neuroprotective effect of NACA treatment following birth asphyxia.

\section{EFFECTS OF THE ISCHEMIC POSTCONDITIONING IN A NEONATAL STROKE MODEL}

doi:10.1136/archdischild-2012-302724.0307

1,2PL Leger, ${ }^{3 P}$ Bonnin, $1,2 \mathrm{~S}$ Renolleau, ${ }^{1,4} \mathrm{O}$ Baud, ${ }^{1} \mathrm{C}$ CharriautMarlangue. ${ }^{1}$ Neurosciences Research Unit, INSERM U676- Paris Diderot University- Paris VII; 2Pediatric Intensive Care Unit, Armand Trousseau Hospital - APHP- UPMC University- Paris VI; ${ }^{3}$ Cardiovascular and Physiology Research Unit, INSERM U965- Lariboisiere HospitalParis Diderot University- Paris VII; ${ }^{4}$ Neonatal Intensive Care Unit, Robert Debré Hospital - APHP, Paris, France

The lack of efficient neuroprotective strategies for neonatal stroke could be ascribed to pathogenic ischemic processes differentiating adults and neonates. In the present study, we tested the hypothesis that ischemic postconditioning applied immediately after ischemia provides neuroprotection in a neonatal stroke model. Neonatal ischemia was generated by permanent occlusion of the left distal middle cerebral artery combined with 50 min of occlusion of both common carotid arteries (CCA) in P7 rats. Postconditioning was performed by repetitive brief release and occlusion ( $30 \mathrm{~s}, 1$ or $5 \mathrm{~min}$ ) of CCA after $50 \mathrm{~min}$ of CCA occlusion. Alternative reperfusion was generated by controlled release of the bilateral CCA occlusion. Blood-flow velocities in the left internal carotid artery were measured using ultrasound imaging with sequential Doppler recordings. Cortical perfusion was measured using laser Doppler. Local cerebral bloodflow was measured by iodo $\left[{ }^{14} \mathrm{C}\right]$ antipyrine autoradiography. None of the procedures of serial mechanical interruptions of blood flow applied at reperfusion induced a reduction of infarct volume after 72 hours. In contrast to adult ischemia, a gradual perfusion was found during early re-flow both in the left internal carotid artery and in the cortical penumbra. No hyperemia was detected on autoradiograms. In addition, vasodilation to hypercapnia remained unchanged. Absence of acute hyperemia during early CCA re-flows and absence of increased cerebrovascular reactivity could at least in part explain the inefficiency of ischemic postconditioning in the developing brain.

\section{TLR3 ACTIVATION INCREASES THE VULNERABILITY OF THE NEONATAL BRAIN TO HYPOXIA-ISCHEMIA}

doi:10.1136/archdischild-2012-302724.0308

C Mallard, L Stridh, X Wang. University of Gothenburg, Gothenburg, Sweden

Background Activation of the innate immune system by bacterial products, via toll-like receptors (TLRs), has been shown to play a role in neonatal hypoxic-ischemic (HI) brain injury in mice. Less is known about how viral products affects neonatal HI. The aim of this study was to investigate the role of a synthetic mimic of double stranded RNA viral products (Poly I:C) in neonatal HI brain injury and whether these effects were dependent on TLR3 via its specific adaptor protein TRIF.

Method Neonatal wildtype (WT) and TRIF knock out (KO) mice were injected with Poly I:C and $14 \mathrm{~h}$ later subjected to unilateral HI $\left(10 \% \mathrm{O}_{2}, 36^{\circ} \mathrm{C}, 50 \mathrm{~min}\right)$. To evaluate brain damage, immunostaining for myelin basic protein (MBP) and microtubule associated protein-2 (MAP-2) were quantitatively analyzed 5 days after Poly I:C/ HI. Cerebral mRNA expression was investigated for IFN- $\beta$, IL-6, IL-1 $\beta$, TNF- $\alpha$, MCP-1, IP-10 and Fas with RT-qPCR.

Results Poly I:C pre-treatment increased HI brain injury in WT mice, which was blocked in TRIF KO mice. Gene expression analyses showed a TRIF specific upregulation of IFN- $\beta$, IL-6, IL-1 $\beta$, TNF$\alpha$, chemokines IP-10 and MCP-1 and the apoptotic mediator Fas.

In summary, the study shows that activation of TLR3 prior to HI increases neonatal brain injury. The sensitizing effect of Poly I:C was associated with release of a range of type I interferons, proinflammatory cytokines and chemokines. The results may indicate that viral infections in the neonate could have great impact on $\mathrm{HI}$ brain injury in the newborn.

\section{TUMOR NECROSIS FACTOR-INDUCIBLE GENE 6 PROTEIN: A NOVEL NEUROPROTECTIVE FACTOR AGAINST INFLAMMATORY DEVELOPMENTAL BRAIN INJURY}

doi:10.1136/archdischild-2012-302724.0309

${ }^{1} \mathrm{~F}$ Bertling, ${ }^{1} \mathrm{~S}$ Prager, ${ }^{1} \mathrm{R}$ Hermann, 'I Bendix, ${ }^{2} \mathrm{HG}$ Wisniewski, ${ }^{1} \mathrm{U}$ FelderhoffMüser, ${ }^{3} \mathrm{M}$ Keller. 'Dept. of Paediatrics I - Neonatology, University Hospital Essen, Essen, Germany, ${ }^{2}$ Dept. of Microbiology, NYU School of Medicine, New York, NY, USA; ${ }^{3}$ Children's Hospital Passau, Passau, Germany

Background and Aims An important factor of developmental brain injury is inflammation. It has been shown that tumor necrosis factor-inducible gene 6 protein (TSG- 6 ) has anti-inflammatory effects in several inflammatory conditions. Nothing is known so far about the role of TSG- 6 in the developing brain, its impact on inflammation and its therapeutic potential.

Methods PCR, Western Blotting and Immunohistochemistry was performed according to standard protocols. Brain hemispheres of untreated Wistar rats (p1-p15) were evaluated under developmental aspects of TSG-6. LPS-treated rats $(0.25 \mathrm{mg} / \mathrm{kg}$ LPS i.p. on p3) were evaluated under pathological aspects of TSG-6. To evaluate whether exogenous rhTSG-6 reduces inflammatory-induced brain injury, 